\title{
Quantitative analysis of fine nano-sized precipitates in low-carbon steels by small angle neutron scattering
}

\author{
Beak Seok Seong • Eunjoo Shin • Shi-Hoon Choi • \\ Yong Choi • Young Soo Han • Kye Hong Lee • Yo Tomota
}

Received: 20 March 2009 / Accepted: 24 February 2010 / Published online: 27 March 2010

(C) The Author(s) 2010. This article is published with open access at Springerlink.com

\begin{abstract}
The small angle neutron scattering (SANS) technique was used to determine the nano-sized precipitates in interstitial free (IF) and low-carbon (LC) steels with a hot-rolling temperature. The real-space direct model fitting method was applied to quantitatively analyze the SANS data. The magnetic and chemical properties of precipitates in the samples were also investigated by separation and comparison of nuclear and magnetic SANS scattering data. The size distribution of precipitates in the IF steel is in good agreement with the microstructure observation using transmission electron microscopy. The results revealed that the IF steel had two types of non-magnetic precipitates, $\mathrm{Ti}_{4} \mathrm{C}_{2} \mathrm{~S}_{2}$ and $\mathrm{TiC}$, with the average size of about $30 \mathrm{~nm}$ in diameter and little difference in chemical composition. In case of commercial LC steel, the fine and large precipitates are identified as $\mathrm{MnS}$ and $\mathrm{Fe}_{3} \mathrm{C}$, respectively. The average size of spherical $\mathrm{MnS}$ precipitates was about $4.8 \mathrm{~nm}$ in radius and the distribution is isotropic. It is likely that the LC steels have almost the same composition and a similar size as precipitates such as $\mathrm{MnS}$ and $\mathrm{Fe}_{3} \mathrm{C}$ with different fin-
\end{abstract}

B.S. Seong $(\varangle) \cdot$ E. Shin $\cdot$ Y.S. Han $\cdot$ K.H. Lee

HANARO Center, Korea Atomic Energy Research Institute,

P.O. Box 105, Yuseong, Daejeon 305-353, Korea

e-mail: bsseong@kaeri.re.kr

\section{S.-H. Choi}

Department of Materials Science and Metallurgical Engineering, Sunchon National University, Jeonnam 540-742, Korea

\section{Y. Choi}

Neutron Beam Application Lab., Sunmoon University, Asan, Chungnam 336-840, Korea

\section{Y. Tomota}

Graduate School of Science and Engineering, Ibaraki University, Nakanarusawa, 316-8511 Hitachi, Japan ishing delivery temperatures. Interestingly, the average size and volume fraction of fine precipitates showed no significantly changes under the different finishing delivery temperatures.

\section{Introduction}

Quantitative analysis of precipitates is very important to understand the relationship between the optimized manufacturing process and the final microstructure, especially for the high formability for automobile body application using various high strength steels [1-3]. There are several methods to analyze the precipitates such as X-ray diffraction (XRD), high resolution transmission electron microscopy (HR-TEM), atom probe field ion microscopy (APFIM) and neutron scattering [2-9]. Among these methods, small angle neutron scattering (SANS) can be a powerful non-destructive method to characterize the quantitative amounts of precipitates in steels. The SANS technique has been widely used to measure the volume fraction and size distribution of the nano-sized structure (1-100 nm) with relatively high accuracy. Furthermore, by the separation and comparison of nuclear and magnetic scattering one can analyze the magnetic and chemical properties of the steel specimen [7]. However, it is necessary to develop the fitting data analysis method, which can quantify the precipitation behavior in steels. In this paper, we suggest the quantitative SANS methods, which adopt the real-space direct model fitting method in poly-dispersed dilute system [2]. The proposed methodology was applied for the quantitative analysis of the nano-sized precipitates of hot-rolled steels. 
Table 1 Chemical

compositions of the low-carbon

(LC) and IF steels

\begin{tabular}{llllllll}
\hline Steels & \multicolumn{6}{l}{ Chemical composition (wt\%) } \\
\cline { 2 - 8 } & $\mathrm{C}$ & $\mathrm{Mn}$ & $\mathrm{P}$ & $\mathrm{S}(\mathrm{ppm})$ & $\mathrm{Sol}-\mathrm{Al}$ & $\mathrm{Ti}(\mathrm{ppm})$ & $\mathrm{N}(\mathrm{ppm})$ \\
\hline LC & 0.020 & 0.20 & 0.011 & 40 & 0.025 & - & 20 \\
IF & 0.003 & 0.10 & 0.012 & 60 & 0.042 & 0.045 & 25 \\
\hline
\end{tabular}

\section{Experimental details}

Low-carbon (LC) steels were used to study the precipitation behavior of the steel with hot-rolling temperature. The LC steel is a modified AISI 1024 grade produced by the POSCO, Korea. The chemical compositions of the steel samples are summarized in Table 1. The ingots of the LC steel were melted in a vacuum and hot rolled to a small slab with a thickness of $30 \mathrm{~mm}$ after a heating to $1,200^{\circ} \mathrm{C}$. After reheating to $1,150^{\circ} \mathrm{C}$ for $120 \mathrm{~min}$, the slab was hot rolled to sheet at the temperature range from 857 to $912^{\circ} \mathrm{C}$ as finishing delivery temperature (FDT) and cooled to $650^{\circ} \mathrm{C}$ corresponding to coiling temperature.

The SANS experiments with a magnetic field of 1.0 Tesla were performed the HANARO Reactor [10]. The wavelength $(\lambda)$ used was $0.508 \mathrm{~nm}$, the sample-to-detector distance was $3.0 \mathrm{~m}$ and the wavelength spread $(\Delta \lambda / \lambda)$ was about $12 \%$. The intensity was obtained in $Q$ values ranging between 0.1 and $1.2 \mathrm{~nm}^{-1}$, corresponding to precipitate sizes of $5 \sim 60 \mathrm{~nm}$ in real space $(Q=4 \pi \sin \theta / \lambda$, where $2 \theta$ is the scattering angle). Two-dimensional scattering patterns collected by the two-dimensional area detectors with an active area of $64.5 \times 64.5 \mathrm{~cm}^{2}$ were averaged to produce a one-dimensional intensity profile. Calibration to absolute SANS cross section was obtained by measurement of standard porous silica in a $1 \mathrm{~mm}$ path-length quartz cell [10]. The data were treated by ILL standard programs [11].

The reliability of quantitative SANS analysis was verified by using a porous silica standard sample and an interstitial free (IF) steel. Porous silica contains mono-dispersed nanosized pores in $\mathrm{SiO}_{2}$ matrix, which is manufactured by National Institute of Standards and Technology (NIST), USA. The IF steel contains fine TiC precipitates less than $60 \mathrm{~nm}$, which was rolled at $900^{\circ} \mathrm{C}$ followed by annealing at $600^{\circ} \mathrm{C}$ for controlling the size and distribution of precipitates.

In order to confirm the distribution of precipitates estimated by quantitative SANS analysis, TEM analysis was performed. Microstructure observation was performed by an optical microscopy (Nikon 2400) and a transmission electron microscopy (TEM, Philips CM1200). Precipitates were analyzed by TEM using the standard replica method. Moreover, energy dispersed spectroscopy (EDS) was employed to identify the precipitates.

\section{SANS data analysis method}

SANS technique has been widely used to characterize the precipitates in the bulk metals and alloys. When a horizontal magnetic field was applied perpendicular to in coming neutron beam in order to align the magnetic moments in the sample, only nuclear scattering occurs in the horizontal plane, while nuclear and magnetic scattering occur in the vertical one. In the case of steel samples, the total SANS cross section, $d \Sigma(Q) / d \Omega$ (where $\Omega$ stands for the solid angle), can be written as the sum of two terms [12]

$d \Sigma(Q) / d \Omega=d \Sigma(Q) / d \Omega_{\mathrm{nucl}}+d \Sigma(Q) / d \Omega_{\mathrm{mag}} \sin ^{2} \alpha$,

where $d \Sigma(Q) / d \Omega_{\text {nucl }}$ and $d \Sigma(Q) / d \Omega_{\text {mag }}$ are the nuclear and magnetic SANS cross section, respectively, and $\alpha$ is the azimuthal angle on the detector plane. The ratio of the vertical to the horizontal SANS components:

$R(Q)=\frac{d \Sigma(Q) / d \Omega_{\mathrm{nucl}}+d \Sigma(Q) / d \Omega_{\mathrm{mag}}}{d \Sigma(Q) / d \Omega_{\mathrm{nucl}}}=1+\frac{(\Delta \rho)_{\mathrm{mag}}^{2}}{(\Delta \rho)_{\mathrm{nucl}}^{2}}$,

is closely related to the composition of the microstructural inhomogeneities. Its dependence on $Q$ implies that precipitates of different size or composition are present in the investigated sample, $(\Delta \rho)^{2}$ being the square difference in neutron scattering length density (nuclear, $(\Delta \rho)_{\text {nucl }}^{2}$, and magnetic $(\Delta \rho)_{\text {mag }}^{2}$, respectively) between the observed nuclear and magnetic precipitates and matrix. In the case of nonmagnetic precipitates, if $(\Delta \rho)_{\mathrm{mag}}^{2}$ is known, then (2) can be used for estimating the nuclear scattering length density of the precipitates from measured scattering curves. Therefore, the assumed chemical composition of precipitates can be estimated by comparing the nuclear scattering length density obtained from measurements to the calculated one. However, when more than one type of precipitates is present which also show different sizes, $R(Q)$ will not be constant, but changes with $Q$. In this case, $(\Delta \rho)_{\text {nucl }}^{2}$ is no longer a constant, but depends on the size of precipitates [7].

Steel is a poly-dispersed dilute system, i.e., the precipitates in the iron matrix have several sizes, shapes and internal structures. Thus the macroscopic nuclear and magnetic 
Table 2 The size and the volume fraction of pores estimated by the Guinier approximation and the direct model fitting method

\begin{tabular}{lllllll}
\hline & $\begin{array}{l}\text { Fitting } Q \\
\text { range }\left[\mathrm{nm}^{-1}\right]\end{array}$ & $\begin{array}{l}R_{g} \\
{[\mathrm{~nm}]}\end{array}$ & $\begin{array}{l}\langle R\rangle \\
{[\mathrm{nm}]}\end{array}$ & $\begin{array}{l}d \Sigma / d \Omega(0) \\
{\left[\mathrm{cm}^{-1}\right]}\end{array}$ & $\begin{array}{l}\text { Number density } \\
{\left[\times 10^{16} / \mathrm{cm}^{3}\right]}\end{array}$ & $\begin{array}{l}\text { Volume fraction } \\
\left\langle V_{f}\right\rangle\end{array}$ \\
\hline NIST (Guinier) & $0.2 \sim 0.4$ & 2.93 & 3.78 & 26.5 & - & - \\
Guinier & $0.2 \sim 0.4$ & 2.94 & 3.80 & - & 4.23 & $9.7 \times 10^{-3}$ \\
Model Fit & $0.05 \sim 1.0$ & 3.14 & 3.95 & - & 3.92 & $8.3 \times 10^{-3}$ \\
Error $(\%)$ & - & 7.2 & 4.4 & - & 7.3 & 14.5 \\
\hline
\end{tabular}

cross section for spherical centers is given by the (3) $[9,13]$.

$\frac{d \Sigma}{d \Omega}(Q)=(\Delta \rho)^{2} \int_{0}^{\infty}\left(\frac{4}{3} \pi R^{3}\right)^{2} N(R) F^{2}(Q, R) d R$

where $N(R) d R$ is the number per unit volume of centers with typical size between $R$ and $R+d R ; F^{2}(Q, R)$ is the form factor of spherical precipitate and $\mathrm{R}$ is the radius of the spherical one. $(\Delta \rho)^{2}$ is the nuclear and magnetic scattering contrast depending on the chemical composition of both the scattering centers (precipitates) and the matrix. When the matrix is magnetized to saturation and the chemical size of the precipitates is assumed to be the same as their magnetic size, the $(\Delta \rho)^{2}$ in (3) can be replaced by (4) [12]:

$(\Delta \rho)^{2}=\left(\Delta \rho_{\text {nucl }}\right)^{2}+\left(\Delta \rho_{\text {mag }}\right)^{2} \sin ^{2} \alpha$

Therefore, the nuclear cross section can be measured at $\alpha=0$, while the sum of nuclear and magnetic cross section is measured at $\alpha=\pi / 2$. Consequently, the magnetic cross section can be calculated by subtracting the data measured at $\alpha=0$ from those obtained at $\alpha=\pi / 2$. When the precipitates are assumed to be non-magnetic, the value of the magnetic scattering length density for the matrix is only equal to the difference in magnetic scattering length density between precipitate and matrix. This means that the volume fraction of the precipitates can be extracted from the magnetic cross section without any information regarding the chemical composition of the precipitates, if the magnetic scattering length density of the matrix is known.

We assume that the size distribution function can be described by a set of one or more log-normal size distributions of spheres per curve;

$N(R)=\sum_{i} N_{0 i} \exp \left[-0.5\left(\frac{\ln (R)-\ln \left(R_{0 i}\right)}{s_{i}}\right)^{2}\right]$

where $N_{0}, R_{0}$ and $s$ are the standard scaling factor, center and width parameters of this distribution type, respectively. The parameters of the log-normal size distributions can be calculated by using a non-linear least-square fitting method [13]. Consequently, the average size, $\langle R\rangle$, and volume fraction, $\left\langle V_{f}\right\rangle$ of precipitates can be estimated from the log-normal size distributions.
The low limit $\left(Q R_{G} \ll 1\right)$ behavior of scattering intensity can be written using the Guinier expression [14] for all spherical type precipitates in dilute system such as steels;

$\frac{d \Sigma}{d \Omega}(Q)=n V^{2}(\Delta \rho)^{2} \exp \left(-Q^{2} R_{G}^{2} / 3\right)$

where $R_{G}$ is the radius of gyration of each individual scatters, $(\Delta \rho)^{2}$ is the scattering contrast, $V$ is the total volume and $n$ is the concentration of scatterers (precipitates). An important feature of this approximation is that $R_{G}$ can be estimated even if $I(Q)$ is known only in arbitrary units.

In the large $Q$ region of scattering, the signal due to the interface between each scatterer and embedding medium dominates the intensity. The Porod law in the ranges of $Q R>4$ can describe this behavior as follows [15]:

$\frac{d \Sigma}{d \Omega}(Q)=2 \pi(\Delta \rho)^{2} S / V Q^{-4}=A_{P} Q^{-4}$

where $S / V$ is the total area of the interface per unit volume of sample and $A_{P}$ is the Porod constant, which is closely related to the scattering contrast factor and the total interface area between precipitates and matrix in steel samples.

\section{Results and discussion}

\subsection{SANS measurement of interstitial free steel}

In the neutron scattering point of view, precipitates in steel act as a poly-dispersed dilute system. In order to verify the current analysis method to quantify the precipitates of steels, two kinds of materials such as porous silica and IF steel were selected as standard and reference samples, respectively. As the standard sample, the porous silica (SIL A4) contains nano-sized pores less than $10 \mathrm{~nm}$ in $\mathrm{SiO}_{2}$ matrix. The scattering length density of the $\mathrm{SiO}_{2}$ matrix is $\rho_{\mathrm{SiO}_{2}}=$ $1.98 \times 10^{11}\left[\mathrm{~cm}^{-2}\right]$ and the scattering contrast constant of pores is $(\Delta \rho)=1.98 \times 10^{11}\left[\mathrm{~cm}^{-2}\right]$. The physical parameters supplied by NIST were listed in Table 2. Figure 1 shows the one-dimensional SANS spectra (circles) and the fitted spectra (line) by using a direct model fitting method for the porous silica sample. The SANS spectra, Fig. 1(a), show two slop regions and one little flat region, which means that the porous silica sample may have two different sizes in pores. 
Fig. 1 Measured (circles) and fitted (line) SANS spectra (a) of porous silica SIL A4 and pore size distribution; (b) estimated by the quantitative SANS analysis

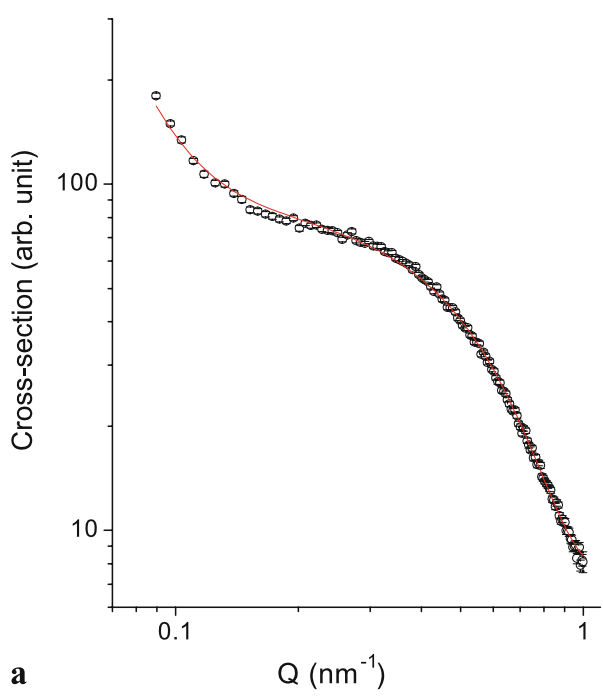

The model fitting of the real-space size distribution from the scattering patterns was performed using a non-linear least-square fitting method. Two log-normal size distributions of spherical pores per curve were considered with a Porod background [1, 15] which was fitted to the $Q$ range between 0.08 and $1.1 \mathrm{~nm}^{-1}$ in (3). It revealed that two types of pores can exit with different sizes. One is fine pores, having an average size of $\sim 3.95 \mathrm{~nm}$ in radius and the other is the larger size. The radius of gyration of the fine pores was calculated using the Guinier approximation [14] in the $Q$ ranges of $0.2 \sim 0.4 \mathrm{~nm}^{-1}$. Figure 1 (b) shows the size distribution of pores obtained by the SANS analysis. The pore radii, number densities and volume fractions calculated by Guinier approximation and the direct model fitting method are summarized in Table 2. The Guinier approximation is in good agreement with NIST results within $\pm 1 \%$ error bound. The direct model fitting approach for small pore sizes are also in good agreement with those of Guinier approximation within average $\pm 7.3 \%$ error bound except for the volume fraction of pores. Since the reason of the increased error in the direct model fitting method is mainly due to the different fitting $Q$ range, the error of SANS analysis can be minimized as the same fitting $Q$ ranges are selected for all investigated samples.

Although quantitative SANS analysis for the porous silica standard sample was successfully satisfied, it is not clear that these results are suitable to apply for the industrial steels due to the complex compositions and various types of precipitates. In this regard, the quantitative SANS analysis combined with the real-space direct model fitting method was used for the analysis of the size and distribution of precipitates in the commercial IF steel.

Figure 2 shows the nuclear SANS cross section (open triangles), the nuclear plus magnetic scattering cross section (open circles) extracted from the 2D-SANS patterns, and the

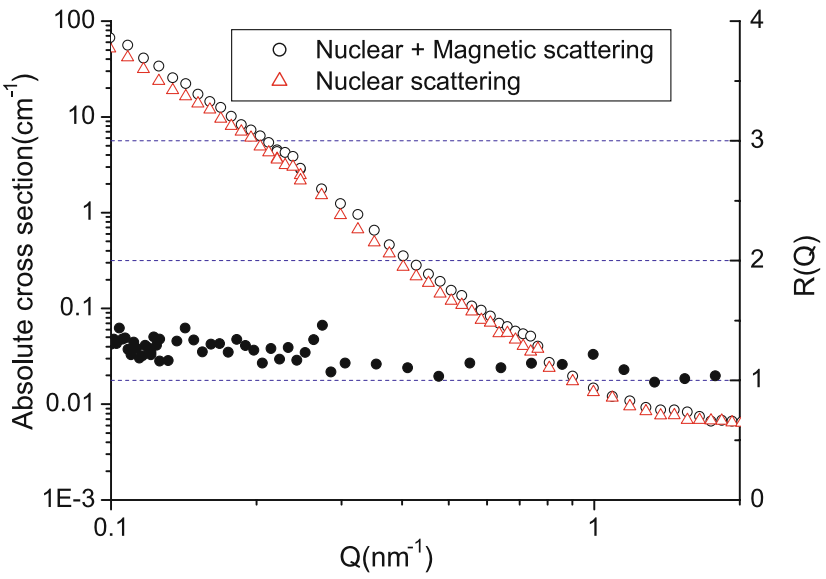

Fig. 2 The nuclear SANS cross section (open triangles) with nuclear plus magnetic scattering cross section (open circles) extracted from the 2D-SANS patterns and the $R(Q)$ values (full circles) for IF steel

$R(Q)$ values (full circles) described by (2) in the IF steel. It shows that the magnetic scattering is not strong over the all $Q$ ranges and the SANS contributions from the magnetic scattering can be negligible in the range of $Q>0.3 \mathrm{~nm}^{-1}$, corresponding to a precipitate size of $<20 \mathrm{~nm}$. The average $R(Q)$ values was 1.29 and it decreases very slowly in the ranges from 0.1 to $0.2 \mathrm{~nm}^{-1}$, then kept nearly constant $(\sim 1.14)$ in the ranges of $Q>0.4 \mathrm{~nm}^{-1}$. This suggests that there is more than one type of non-magnetic fine precipitate such as $\mathrm{Ti}_{4} \mathrm{C}_{2} \mathrm{~S}_{2}$ and $\mathrm{TiC}$ and their chemical compositions are little different from stoichiometry. Since the calculated $R(q)$ value is about 1.5 for non-magnetic TiC precipitates, the smaller $R(Q)$ value may be due to the changes of chemical compositions of the precipitates and matrix. The magnetic scattering contribution to SANS spectra can be negligible.

The nuclear scattering length density, $(\Delta \rho)_{\mathrm{TiC}}=6.4 \times$ $10^{10} \mathrm{~cm}^{-2}$, was calculated with the assumption that the 
fine precipitates less than $50 \mathrm{~nm}$ were TiC. The model fitting was performed using a non-linear least-square fitting method. Two log-normal size distributions of spherical precipitates per curve were considered with the Porod background, which were fitted to the $Q$ range between 0.15 and $2.0 \mathrm{~nm}^{-1}$ in (3).

In order to verify the SANS results and determine the statistical data of the size distribution of precipitates, the microstructure was analyzed by TEM. The TEM samples were prepared by the standard carbon replica method. The precipitates of the IF steels are known as $\mathrm{TiC}, \mathrm{Ti}_{4} \mathrm{C}_{2} \mathrm{~S}_{2}$, and TiS [3]. Figure 3 shows the statistical data about the size distribution of the precipitates in the IF steel estimated by the quantitative SANS analysis (line) compared with TEM analysis (bar). As shown in Fig. 3, the average sizes of precipitates calculated by SANS and TEM analysis were $29.1 \mathrm{~nm}$ and $31.8 \mathrm{~nm}$, respectively. A trend of size distribu-

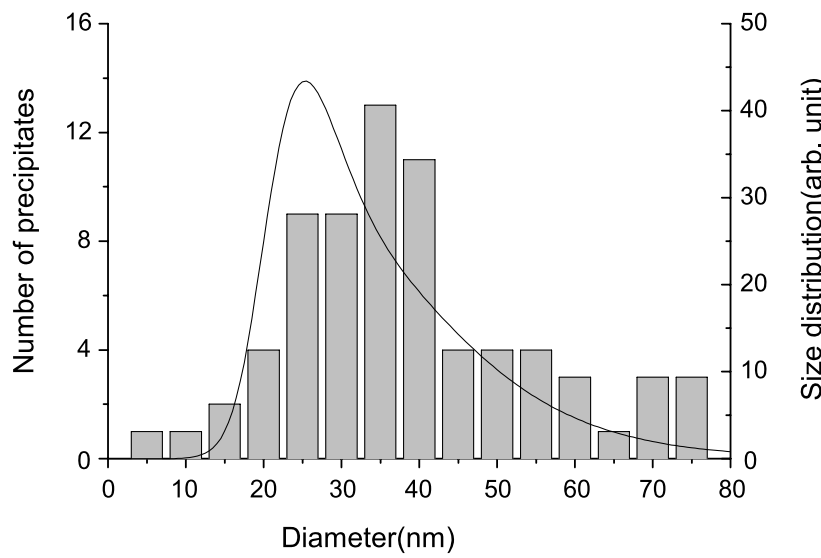

Fig. 3 The comparison of size distributions obtained by SANS (line) with TEM analysis (bar)
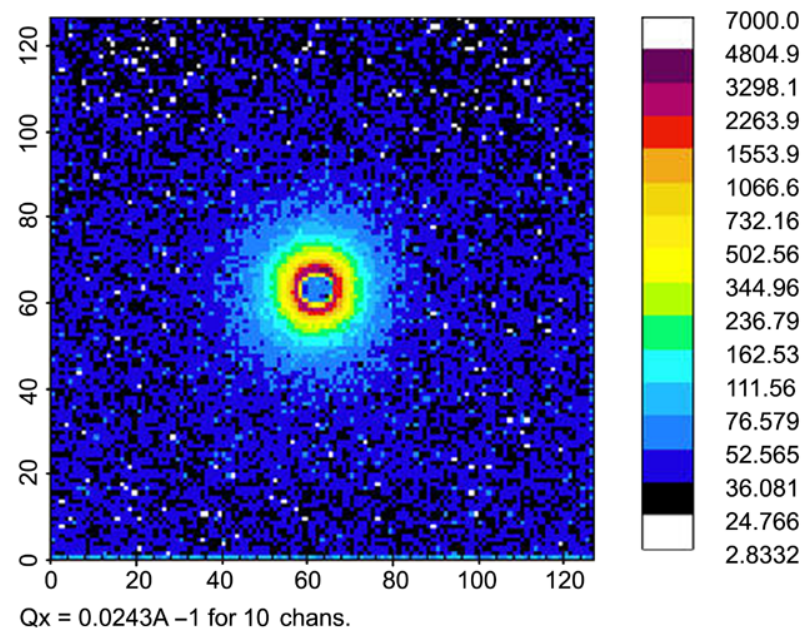

a $\mathrm{FDT} 875^{\circ} \mathrm{C}$ tion estimated by SANS analysis is almost similar to the one by TEM analysis. The good agreements of the two methods support that the SANS technique might be a promising method to measure the volume fraction and size distribution of precipitate in bulk steel samples, although the SANS obtains the statistical information and TEM can be localized.

\subsection{SANS measurement of low-carbon steel}

Figure 4 shows the examples of two-dimensional SANS spectra obtained with the magnetic field of $1.0 \mathrm{~T}$ for commercial low-carbon (LC) steels for different hot-rolling temperatures, FDT $875^{\circ} \mathrm{C}$ and $912^{\circ} \mathrm{C}$. As shown in Fig. 4, the patterns of all samples show a nearly isotropic feature. In order to investigate the effect of the magnetic scattering on the SANS patterns, the nuclear scattering contributions extracted from the 2D-SANS patterns are compared with nuclear plus magnetic scattering contributions. Figure 5 shows the magnetic scattering effect on SANS data of a commercial LC steels with different hot rolling temperature. The $R(Q)$ values were included in Fig. 5 to investigate the variations of chemical composition and precipitation of the precipitates. As shown in Fig. 5, the magnetic scattering of all samples is not strong over all $Q$ ranges. The contributions from the magnetic scattering can be negligible in the range of $Q>0.3 \mathrm{~nm}^{-1}$, corresponding to a precipitate size of $<20 \mathrm{~nm}$. $R(Q)$ values for all samples are nearly constant $(\sim 1.2)$. It means that the fine $\mathrm{MnS}$ precipitates keep a homogeneous chemical composition during finishing delivery temperature and the magnetic scattering contrasts between precipitates and matrix can be negligible.

The intensity of the SANS spectra showed very small differences over all the measured $Q$ ranges in the samples according to the finishing delivery temperature as shown in

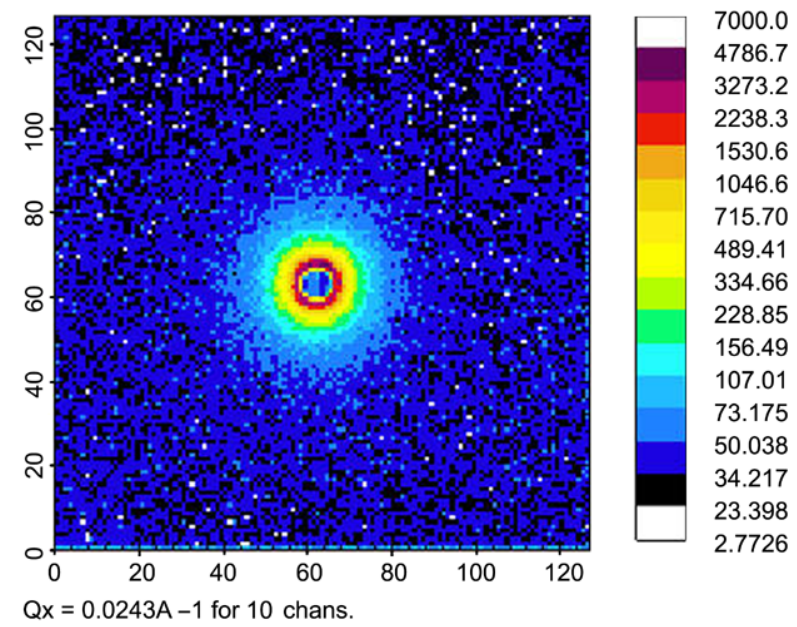

b FDT $912^{\circ} \mathrm{C}$

Fig. 4 Two-dimensional SANS spectra of the LC steel with the two different finishing delivery temperatures: (a) FDT $875^{\circ} \mathrm{C}$; (b) FDT $912^{\circ} \mathrm{C}$ 
Fig. 5 Magnetic scattering effect on SANS spectra of the LC steel with the two different finishing delivery temperatures: (a) FDT $875^{\circ} \mathrm{C}$; (b) FDT $912^{\circ} \mathrm{C}$
Fig. 6 Measured (circles) and fitted (line) nuclear SANS cross section for the LC steel with the two different finishing delivery temperatures: (a) FDT $875^{\circ} \mathrm{C}$; (b) FDT $912^{\circ} \mathrm{C}$
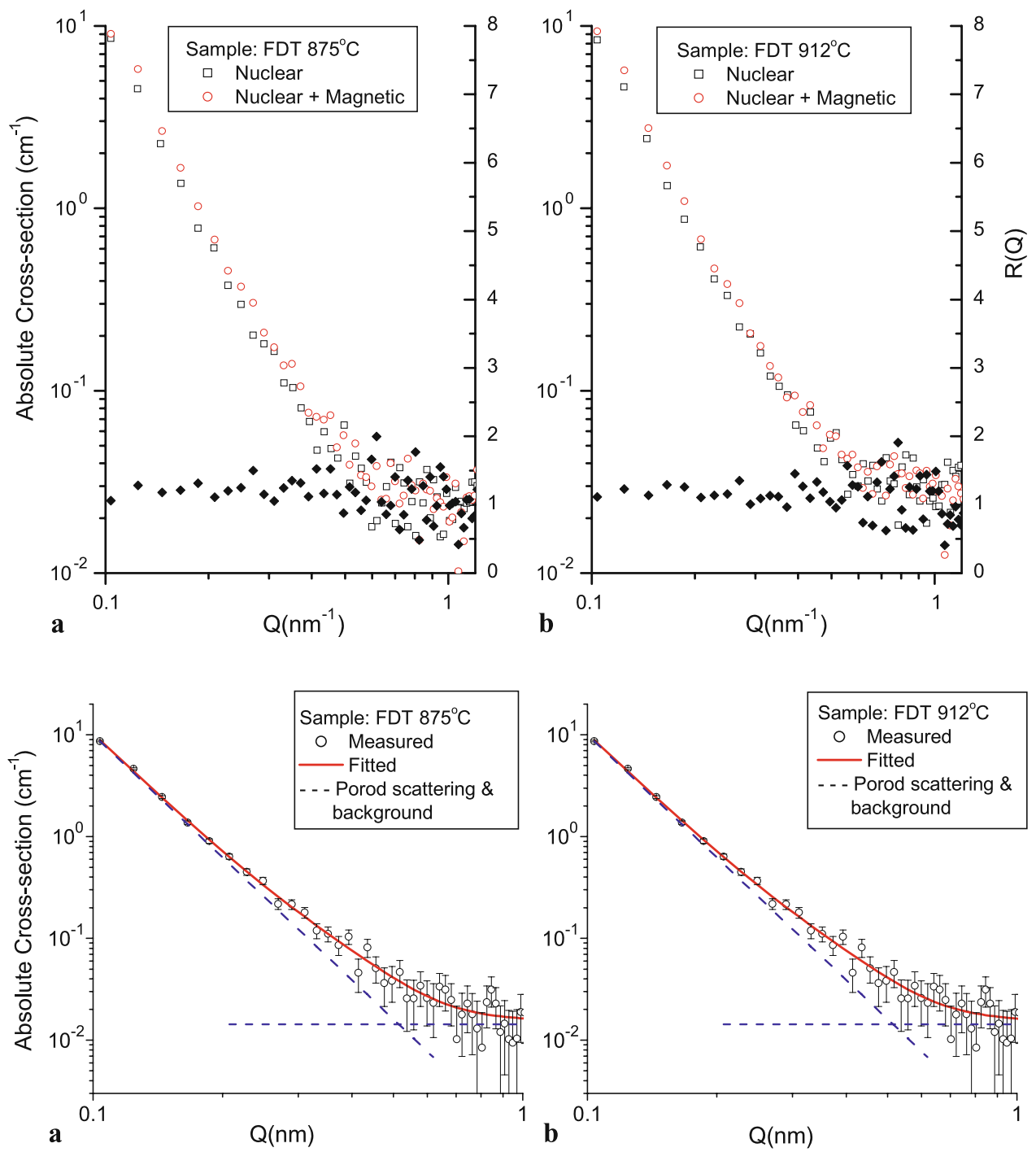

Fig. 5. It means that the size and distribution of the precipitates have no significantly changes for different hot-rolling temperature. The intensities followed $\sim A_{p} Q^{-4}$ in the $Q$ range of $<0.2 \mathrm{~nm}^{-1}$, expressed in (7). The Porod constant $\left(A_{p}\right)$ is proportional to the total area of the interface between the precipitates and matrix. The intensity in the $Q$ range of $>1.0 \mathrm{~nm}^{-1}$ became close to the background level. The scattering length density is related to the possible fine scattering materials. The nuclear scattering length densities of $\mathrm{MnS}$ and $\mathrm{Fe}_{3} \mathrm{C}$ precipitates contributed to SANS intensities in the $Q$ range of $>0.2 \mathrm{~nm}^{-1}$ are $\rho_{\mathrm{MnS}}=-0.25 \times 10^{10} \mathrm{~cm}^{-2}$ and $\rho_{\mathrm{Fe}_{3} \mathrm{C}}=9.0 \times 10^{10} \mathrm{~cm}^{-2}$, respectively. The squared scattering contrast between the pure iron matrix and $\mathrm{MnS}$ precipitates is higher by factors 60 than that for $\mathrm{Fe}_{3} \mathrm{C}$ precipitates. It suggests that the SANS intensity was mainly attributed to the fine $\mathrm{MnS}$ precipitates.

A model fitting of the real-space size distribution to the SANS spectra was performed by using a non-linear leastsquare method. A log-normal size distribution of the pre- cipitates per curve was considered and fitted to the $Q$ range between 0.1 and $1.2 \mathrm{~nm}^{-1}$ in (3). The Porod background, $I(Q) \sim A_{p} Q^{-4}+$ bkg (background), was used for model fitting. The Porod constant $\left(A_{p}\right)$ is closely related to the scattering contrast factor and total interface area between precipitates and matrix. The bkg is proportional to the measured incoherent scatterings due to mostly concentrations of nuclei and the contributions of small defects such as vacancies.

Figure 6 shows the measured nuclear SANS spectra and the fitted spectra for the samples with different hotrolling temperature. Open circles are corresponding to the observed intensities and the solid line represents the fitted ones. Dashed line denotes Porod scattering and background. Even though the spectra are very weak and have poor statistics in the range of $>0.5 \mathrm{~nm}^{-1}$, the fitted spectra are in good agreement with the measured ones. As model fitting results, Porod constants $\left(A_{p}\right)$ and backgrounds of the samples were obtained with almost the same values, $1.04 \times 10^{25}\left[\mathrm{~cm}^{-5}\right]$ 
Fig. 7 The number distribution, volume fraction and average size of the precipitates for different finishing delivery temperatures calculated by quantitative SANS analysis
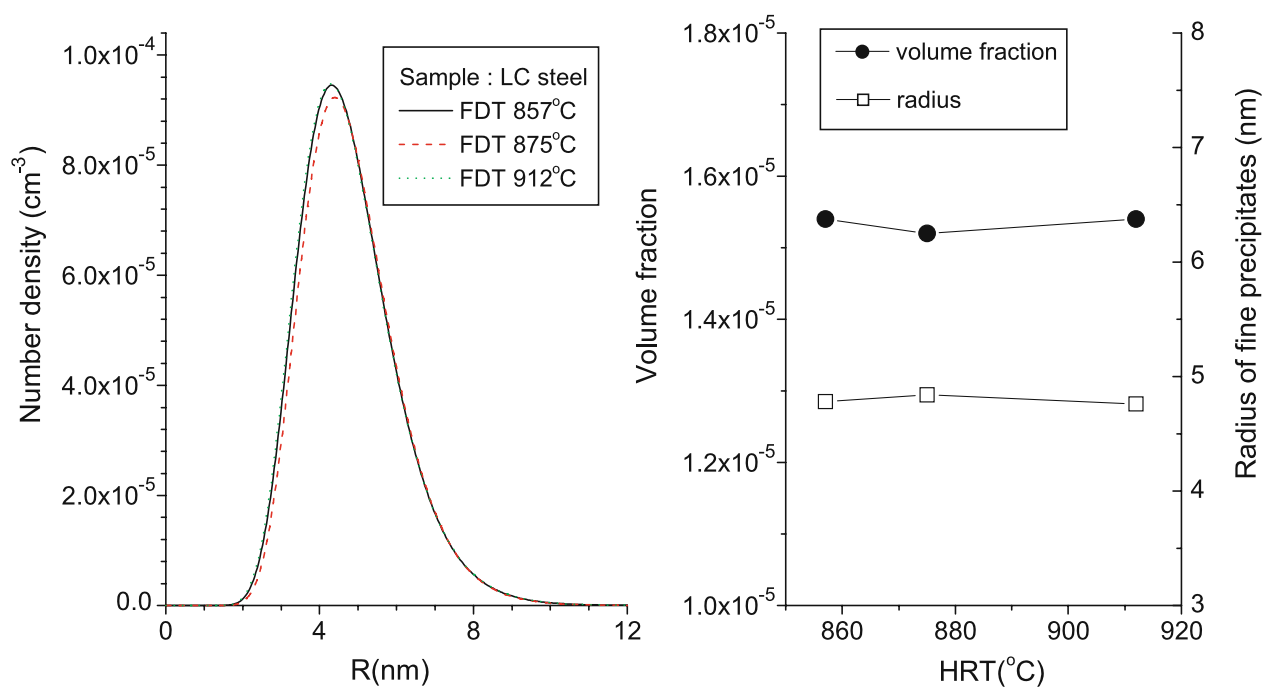

and $0.0143\left[\mathrm{~cm}^{-1}\right]$, respectively. This suggests that all samples have a similar type of large precipitates like cementite phases and have almost the same compositions of precipitates and interface area between matrix and precipitates. It is well known that the cementite $\left(\mathrm{Fe}_{3} \mathrm{C}\right)$ is in fact ferromagnetic. The average magnetic moment of cementite can be close to that of a typical steel matrix, leading to very low magnetic scattering contrast as shown in Fig. 5 in the rage of $Q<0.2 \mathrm{~nm}^{-1}$. This is due to the high iron content and relatively low carbon to iron ratio of cementites. Figure 7 shows the number distribution, volume fraction and average size of the precipitates calculated by quantitative SANS analysis as a function of the finishing delivery temperature. The results show that the average size and volume fraction of the fine $\mathrm{MnS}$ precipitates showed no significantly changes, even if the finishing delivery temperature was changed. The average size of fine $\mathrm{MnS}$ spherical precipitates for all samples was about $4.8 \mathrm{~nm}$. The LC steels also had the similar size dispersions of the precipitates having almost the same compositions with finishing delivery temperature.

\section{Summary}

A quantitative SANS analysis combined with a real-space direct model fitting method was proposed, verified and applied to quantitatively analyze the nano-sized precipitates in commercial industrial samples. In case of the interstitial free (IF) steel, there are two types of non-magnetic precipitates less than $50 \mathrm{~nm}$ such as $\mathrm{Ti}_{4} \mathrm{C}_{2} \mathrm{~S}_{2}$ and $\mathrm{TiC}$, indicating that the chemical compositions are little different each other. The magnetic scattering contribution to SANS spectra can be negligible. Average sizes of precipitates of the IF steel calculated by SANS were $29.1 \mathrm{~nm}$ in diameter and it is well agreement with TEM analysis. In case of low-carbon (LC) steel, the large precipitates in the LC steel are likely to be cementite which shows larger than $50 \mathrm{~nm}$ size in diameter and have almost same compositions and interface area. The results revealed that the size distribution of the fine precipitates is isotropic. The average size $(\sim 4.8 \mathrm{~nm})$ and volume fraction $\left(\sim 1.5 \times 10^{-5}\right)$ of fine MnS precipitates had no significantly changes with the hot-rolling temperature. The chemical composition of the large and the fine precipitates in the LC are homogeneous and have similar size dispersions as a function of hot-rolling temperatures.

Acknowledgements This work was supported by Nuclear Research \& Development Program of the Korea Science and Engineering Foundation (KOSEF) grant funded by the Korean government (MEST). The authors thank Dr. Chuck Woo for helpful discussions and advices.

Open Access This article is distributed under the terms of the Creative Commons Attribution Noncommercial License which permits any noncommercial use, distribution, and reproduction in any medium, provided the original author(s) and source are credited.

\section{References}

1. Y.R. Cho, S.I. Kim, B.S. Seong, Iron Steel Technol. 1, 46 (2004)

2. B.S. Seong, Ph.D. Thesis. Ibaraki University, Hitachi, Japan (2008)

3. E. Shin, B.S. Seong, H.-J. Kang, M.-Y. Huh, Z. Met.kd. 94, 1234 (2003)

4. Z. Guo, W. Sha, J. Appl. Cryst. 37, 325 (2004)

5. V.K. Vasudevan, S.J. Kim, C.M. Waymann, Metall. Trans. A 21A, 2655 (1990)

6. J. Mittra, G.K. Dey, D. Den, A.K. Patra, S. Mazumder, P.K. De, Scr. Mater 51, 349 (2004)

7. M. Bischof, P. Staron, D. Caliskanoglu, H. Leitner, C. Scheu, H. Clemens, Mater. Sci. Eng. A 472, 148 (2008)

8. M. Ojima, M. Ohnuma, J. Suzuki, S. Ueta, S. Narita, T. Shimizu, Y. Tomota, Scr. Mater. (2008) 
9. E. Shin, B.S. Seong, Y.S. Han, K.P. Hong, C.H. Lee, H.-J. Kang, J. Appl. Cryst. 36, 624 (2003)

10. B.S. Seong, Y.S. Han, C.H. Lee, J.S. Lee, K.P. Hong, K.N. Park, H.J. Kim, Appl. Phys. A 74, S201 (2002)

11. R. Ghosh, S.U. Egelhaaf, A.R. Rennie, ILL report, ILL06GH05T (2006)

12. G.E. Bacon, Neutron Diffraction, 3rd edn. (Clarendon, Oxford, 1975)
13. J. Kohbrecher, SASFITTCL (c), version 0.2 alpha. PSI. Germany (1999)

14. L.A. Feigin, D.J. Svergun, Small Angle X-ray and Neutron Scattering (Plenum, New York, 1987)

15. G. Porod, in Small Angle X-ray Scattering, ed. by O. Glatter, O. Krathy (Academic Press, London, 1982) 